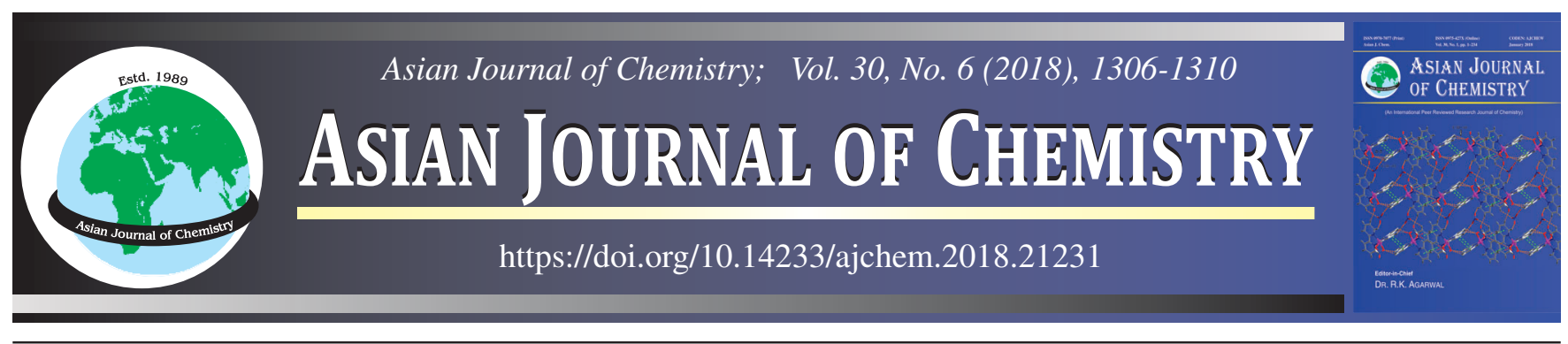

\title{
Entrapment of Natural Flavonoids for Microparticulate Drug Delivery System for Hydrophobic Drug Rutin and Hesperidin
}

\section{Ripunjoy Bordoloi ${ }^{1, *}$, Pulak Deb ${ }^{1}$, Barnali Gogol ${ }^{2}$, Himakshi Baishya ${ }^{2}$ and Parthajyoti Gogoi ${ }^{2}$}

${ }^{1}$ Girijananda Chowdhury Institute of Pharmaceutical Sciences, Azara Hathkhowapara-781 017, India

${ }^{2}$ Regional Drugs Testing Laboratory, Six Mile Guwahati-781 022, India

*Corresponding author: E-mail: ripun.bordoloi@gmail.com

Received: 15 January 2018;

Accepted: 14 February 2018;

Published online: 30 April 2018;

AJC-18884

The aim of this study was to investigate importance of polymeric micro particulate drug delivery system in pharmaceutical field for
modifying the drug profile of poorly water-soluble drug rutin and hesperidin. In this study sodium alginate is used as a polymer for
entrapping the model drug, which involved the method of ionic cross linking- process. For physico-chemical characterization of the
formulations we have studied in vitro drug release drug entrapment efficiency, Fourier transforms infrared spectroscopy (FTIR), scanning
electron microscopy (SEM) and differential scanning calorimetric (DSC). In case of hesperidin FH1 is having the minimum drug entrapment
efficiency of approximately $69.46 \%$ whereas the entrapment efficiency of FH5 shows an approximate entrapment of $74.28 \%$. Drug:sodium
alginate ratio of $1: 2$ shows an approximate entrapment of $74.28 \%$. In case of hesperidin FH 2 is having the minimum drug entrapment
efficiency of approximately $71.00 \%$ whereas the entrapment efficiency of FH 2 shows an approximate entrapment of $75.7 \%$. This could
be revealed as the increase in polymer concentration has significant effect on the drug entrapment efficiency. Drug:sodium alginate ratio
of $1: 1.2$ shows an approximate entrapment of $75.7 \%$. The FTIR analysis of drug, polymers and the formulations indicated the compatibility
of the drug with the polymers. The scanning electron microscopy was used to confirm the smoothness of microspheres formulated. The
results of the dissolution study indicated that with the increase in the concentration of sodium alginate in the matrix the amount of drug
release appreciably decreased and became steady. The drug-polymer interaction in the microsphere was studied by using differential
scanning calorimetry. The release kinetics of the prepared batches of formulation was also studied and was found that the prepared
batches followed Higuchi and Krosmayer Peppas release mechanism. The findings of the study suggest that the microsphere formulations
is a promising carrier for flavanoids delivery and can be considered as a favourable oral controlled release dosage form for hydrophobic
drug rutin and hesperidin.

Keywords: Rutin, Hesperidin, Flavonoid, Microsphere.

ᄂ _ - _ - - _ - - _ - - - - - - - - - - - - - - - - - - - - - - - -

\section{INTRODUCTION}

Researchers have treasured the potential benefits of micro technology in providing vast improveaments in drug delivery. There are several approaches for delivering a drug to the systemic circulation to get the desired response of a drug substance. For a drug to show its specific action, the drug has to reach the target site at a specific amount and at the defined rate. Generally a drug delivery system can be categorized into the conventional drug delivery systems and the novel and targeted drug delivery systems [1]. The novel and targeted drug delivery systems can be used to overcome the drawbacks or limitations of conventional dosage form which are proving to be more successful and thereby enhancing the patient acceptability. The recent studies have indicated that micro particulate drug delivery systems are suitable for achieving controlled or delayed release oral formulations, targeting a specific site in the body, protecting drug molecule, altering surface properties. The release pattern of drug from micro particles relies on a variety of factors which includes the carrier used to form the microparticles and the amount of drug contained in them. As a whole the micro particulate drug delivery systems provide various opportunities for designing sustained release oral formulations, thus enhancing the opportunity for the future pharmaceutical development of dosage form [2]. Microparticulate drug delivery system is promising method for future drug delivery of several drugs including xenobiotics to enhance their bioavailability patterns. Thus this form of drug delivery system can be promising aspects for delivery of drugs for targeting its action and also for sustaining its release at some case which can be beneficial for treatment of several life threatening diseases. In drug delivery systembiodegradable polymers 
has developed in various fields. Among them two cost effective polymers namely sodium alginate and chitosan are commonly and widely used in pharmaceutical industry $[1,2]$.

An immunomodulator is defined as a substance, biological or synthetic, which can stimulate, suppress or modulate any of the components of the immune system including both innate and adaptive arms of the immune response. Two types of immunomodulators are there which is based on their effect, immune suppressants and immune stimulators. The nonspecific activation of the function and efficiency of macrophages, granulocytes, complement, natural killer cells and lymphocytes are the main components of immunomodulation. These nonspecific effects give protection against different pathogens and constitute an alternative to conventional chemotherapy [3].

Flavonoids are a class of natural compounds which possesses a strong immune modulatory action on the living cell. Almost all the available flavonoids possess a strong action which in turn potentiates the immune system. In spite of possessing immune stimulant activity, the flavonoids also possess other activity like antioxidant, anti-inflammatory, anticancer and some of them have cardiac stimulant action [4].

Rutin is a flavonol, abundantly found naturally in plants. It is a vital nutritional component of food stuff. The name 'rutin' comes from the plant Ruta graveolens, which also contains rutin. Chemically it is a glycoside comprising of flavonolic aglycone quercetin along with disaccharide rutinose. It has demonstrated a number of pharmacological activities, including antioxidant, cytoprotective, vasoprotective, anticarcinogenic, neuroprotective and cardioprotective activities [5,6].

Hesperidin is a flavanone glycoside found in citrus fruits. Its name is derived from the word "hesperidium", the kind of fruit produced by citrus trees. Various preliminary studies reveal novel pharmaceutical properties, none of which has been confirmed as applicable to humans. Hesperidin reduced cholesterol and blood pressure. Hesperidin is also a potential sedative, possibly acting through opioid or adenosine receptors. Hesperidin exhibited pronounced anticancer activity against some selected human carcinoma cell lines [7].

The main aim of this study is to develop an efficient drug delivery method to increase the solubility of rutin and hesperidin. In the present research work rutin and hesperidin microparticulate system has been prepared by ionic gelation method for sustain release thereby reducing the systemic toxicity and dosing interval and to propose the possible release mechanism of the loaded flavonoids from the microparticulated system through kinetic evaluation of in vitro release data. Physicochemical characterization of the formulations has been done by in vitro drug release study, scanning electron microscopy (SEM), differential scanning calorimetry (DSC), Fourier transform infrared spectroscopy (FT-IR).

\section{EXPERIMENTAL}

The drugs rutin and hesperidin were purchased from Otto Chemicals Princess Street Mumbai. Sodium alginate was purchased from Sigma Aldrich, India. The other entire reagent used was of analytical grade.

\section{Formulation studies}

Preparation of rutin and hesperidin micro particles: The micro particle containing the drug was prepared by ionic gelation technique. This method involves the preparation of the polymeric solution of sodium alginate in distilled water. The required amount of drug was then added to the warm polymeric solution with constant agitation. In another beaker, $5 \%$ solution of calcium chloride was dissolved which acts as the cross linking agent. The drug polymeric solution mixture was then added to this solution drop-wise through a $0.45 \mathrm{~mm}$ syringe needle at a dropping rate of $1.0 \mathrm{~mL} / \mathrm{min}$. In order to facilitate the proper formation of micro particles, the solution of cross linking agents was kept under constant stirring at $200 \mathrm{rpm}$. Finally the micro particles were filtered, dried, collected and further evaluation parameters were carried out [8].

Determination of encapsulation efficiency [9]: Entrapment efficiency (EE) of drug loaded microparticles was determined i.e. by calculating the amount of un-entrapped drug, which was performed by cooling centrifugation (Remi, C-24DL) of the microparticles at 10,000 rpm for $30 \mathrm{~min}$. The supernatant liquid was collected separately. The separated particles were washed with distilled water and the washings were mixed with supernatant liquid and again the solutions were recentrifuged. The supernatant liquid then evaluated spectrophotometrically with suitable dilution and filtration.

$$
\mathrm{EE}(\%)=\frac{\text { Drug inital }- \text { Drug supernatant }}{\text { Drug inital }} \times 100
$$

in vitro Release study [10]: The in vitro release study of the formulation was carried out by using dissolution apparatus of rotating paddle type (USP Type II). The dissolution fluid for this purpose was phosphate buffer $\mathrm{pH} 6.8$ as a representative of intestinal fluids. The samples were placed in each compartment containing the dissolution medium which was maintained at $37^{\circ} \mathrm{C}$ and was stirred at $100 \mathrm{rpm}$. After certain time intervals, samples were drawn out and fresh fluid was added in order to maintain the sink condition. Finally the samples were filtered and dilutions if necessary were made and the amount of drug was measured spectrophotometrically against the specified blank solution.

Drug release kinetic studies [11]: For the characterization of the release kinetics studies and to determine the release mechanism of drug, the results of in vitro studies were fitted with several kinetics models as follows.

\section{Zero order rate equation:}

$$
\mathrm{Q}_{\mathrm{t}}=\mathrm{Q}_{0}+\mathrm{K}_{0} \mathrm{t}
$$

where $Q_{t}$ is the amount of drug dissolved in time $t, Q_{0}$ is initial amount of drug in solution and $\mathrm{K}_{0}$ is zero order release constant.

\section{First order rate equation:}

$$
\log C=\log C_{0}-K_{t} / 2.303
$$

where $\mathrm{K}_{0}$ is the initial concentration of drug, $\mathrm{K}$ is first order release constant and $\mathrm{t}$ is time.

\section{Higuchi's model:}

$$
\mathrm{Q}=\mathrm{K}_{\mathrm{H}} \mathrm{t}^{1 / 2}
$$

where $\mathrm{Q}$ is the amount of drug released in time t per unit area, $\mathrm{K}_{\mathrm{H}}$ is Higuchi dissolution constant. 
To find out the mechanism of drug release, the in vitro release data of all niosomal formulations were fitted into Korsmeyer and Peppas equation:

$$
\mathrm{M}_{\mathrm{t}} / \mathrm{M}_{\infty}=\mathrm{Kt}^{\mathrm{n}}
$$

where $M_{t} / M_{\infty}$ is a fraction of drug release at time $t, K$ is the release rate constant and $\mathrm{n}$ is the release exponent. The value of exponent (n) indicates the mechanism of drug release (Table-1).

\begin{tabular}{|c|c|}
\hline & $\begin{array}{c}\text { TABLE-1 } \\
\text { VALUE OF 'n' AND CORRESPONDING } \\
\text { MECHANISM OF DRUG RELEASE }\end{array}$ \\
\hline $\begin{array}{l}\text { Value } \\
\text { of 'n' }\end{array}$ & Mechanism of drug release \\
\hline $\mathrm{n}=0.5$ & Case-I (Fickian) diffusion or square root of time kinetics \\
\hline $0.5<\mathrm{n}<1$ & Anomalous (non-Fickian) diffusion \\
\hline $\mathrm{n}=1$ & Case-II transport/relaxation controlled release \\
\hline$n>1$ & Super Case-II transport \\
\hline
\end{tabular}

Imaging through high resolution digital scanning microscope: The images of the microspheres were taken using high resolution scanning microscope. Randomly chosen microparticles were taken to measure their individual shape and morphology. Microparticles were visualized under $30 \times$ magnification.

Scanning electron microscope (SEM) analysis: The surface morphology and size distribution of prepared micro particles were determined by using scanning electron microscopy (JEOL, JEM 2100) under high resolution electron microscope with an acceleration voltage of $80 \mathrm{KV}$. For the observation of morphology a drop of the sample was transferred into the copper mesh grids. After the sample was adsorbed (about 15-20 min), the staining dye (potassium phosphotungstate) was dripped onto the film. The staining time was about 1-2 min. After drying the copper mesh grids, the surface topography of the niosomes was then visualized using a scanning electron microscope.

Particles size analysis: Particle size of the prepared micro particles was determined using optical microscopy technique. A microscope is fitted with a stage micrometer and desired magnification was adjusted to calculate the particle size of the prepared micro particles.

Drug compatibility study [12,13]: The drug compatibility study of the physical mixture of rutin and sodim alginate, hesperidin and sodium alginate and also for the plant extract were performed by FTIR spectroscopy and DSC analysis.

FT-IR spectroscopy: Drug compatibility with other excipients was studied using, FT-IR spectrophotometer (Bruker 10059736, model alpha, Germany). Each drug-excipient samples were scanned from 4000 to $400 \mathrm{~cm}^{-1}$ to evaluate the physical stability and compatibility of the drugs with the excipient. The FTIR analysis was carried out for pure drug. The spectra obtained were compared with the pure drug spectra.

Differential scanning calorimetry (DSC) study [14]: DSC thermograms of pure rutin and hesperidin and also their mixtures with excipients were recorded using DSC instrument. About $5 \mathrm{mg}$ of sample was placed on the $50 \mu \mathrm{L}$ of aluminium pan in a hermetically sealed condition. The measurement was performed in a nitrogen atmosphere $(20 \mathrm{~mL} / \mathrm{min})$ between $\mathrm{DSC}$ tempera-tures at 50 to $270{ }^{\circ} \mathrm{C}$ at a heating rate of $10^{\circ} \mathrm{C} / \mathrm{min}$. An empty sealed pan was used as reference.

\section{RESULTS AND DISCUSSION}

\section{Drug excipient compatibility study}

FTIR analysis: FTIR studies indicate three bands present in the rutin spectrum, due to $\mathrm{O}-\mathrm{H}, \mathrm{C}-\mathrm{H}, \mathrm{C}=\mathrm{O}$ linkages, respectively. The characteristic peak of the carbonyl group at 1651.24 $\mathrm{cm}^{-1}$; a band of peaks at 3323.78 and $3193.64 \mathrm{~cm}^{-1}$ owing hydroxyl groups; and peaks at 1284.31 and $1157.47 \mathrm{~cm}^{-1}$ owing to asymmetrical and symmetrical stretching of the C-O-C system (present in the oxathiolane ring). The presence of these peaks confirms that the drug is pure rutin. From the IR spectrum of hesperidin the following peaks of $\mathrm{C}-\mathrm{H}$ stretching, $-\mathrm{OH}$ stretching, $\mathrm{C}=\mathrm{C}$ stretching and $\mathrm{C}-\mathrm{O}$ stretching were found. All these spectrum of pure drugs and excipient was compared with the standard as given in the pharmacopoeias and the comparison was found to be satisfactory. The characteristic absorption peak of the carbonyl group at $1651.24 \mathrm{~cm}^{-1}$; also a band of peaks at 3323.78 and $3193.64 \mathrm{~cm}^{-1}$ of hydroxyl groups and peaks at 1284.31 and $1157.47 \mathrm{~cm}^{-1}$ owing to asymmetrical and symmetrical stretching of the $\mathrm{C}-\mathrm{O}-\mathrm{C}$ system (present in the oxathiolane ring) were appear in the spectra. The identical peaks of $\mathrm{C}-\mathrm{H}, \mathrm{C}=\mathrm{O}$ and $\mathrm{O}-\mathrm{H}$ stretching were also appeared in the spectra of rutin, alginate physical mixtures. In sodium alginate some distinct peaks such as carboxyl group showed strong absorption bands at $1606.43 \mathrm{~cm}^{-1}$ due to carboxyl anions asymmetric and symmetric stretching vibrations disappearing or becoming weak in the micro particle formulations which have shown the evidence of multi-interactions (hydrogen binding and electrostatic interaction) among sodium alginate molecules supporting the previous works [15].These observations indicated that no chemical interactions between the drug and the excipient. The obtained spectrum was compared with the pure drug and no shifting of functional group was found in it, with this we can concluded that the drug is compatible with other excipients.

DSC analysis: The DSC thermogram of the pure drug rutin and hesperidin was found out (Fig. 1) and was compared with the standard. There was no any change of the thermogram when compared with the standard thus we can conclude that the drug obtained are pure.

DSC scans of pure Hesperidin showed an endothermic peak at $259^{\circ} \mathrm{C}$, corresponding to the melting point of the compound. Physical mixture of the drug and polymer, in a 1:1 molar ratio, also exhibited the endothermic peak associated with hesperidin. This suggests that there is no any interaction of drug along with the polymers used in preparing the formulation (Fig. 1).

Drug entrapment efficiency: The drug loaded microsphere was taken in an appropriate amount and was dissolved in phosphate buffer $\mathrm{pH}$ 6.8. The mixture was than centrifuged at $200 \mathrm{rpm}$ for $30 \mathrm{~min}$. The supernated liquid was collected and dilution if necessary was made and was measured spectrophotometrically against appropriate blank solution. All the microparticulate formulations were evaluated for drug entrapment efficiency and the results are given in Table- 2 which shows that the entrapment efficiency was affected by the sodium alginate concentration which reflects that with the increase 


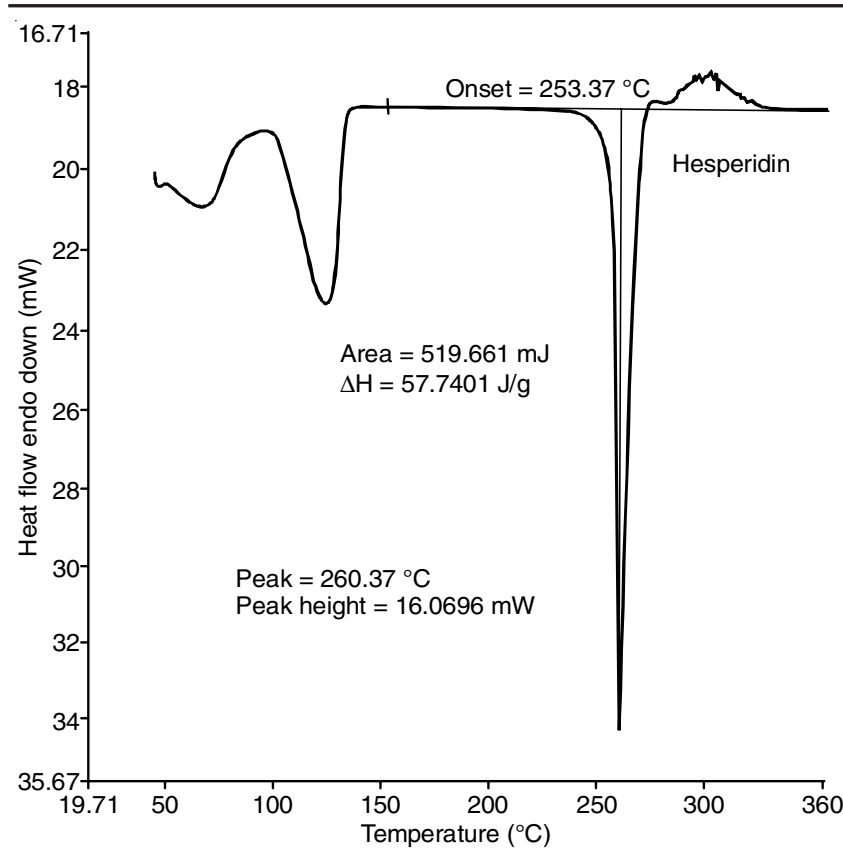

Fig. 1. DSC curves of formulation

in polymer ratio the drug entrapment in the polymer matrix get increased. In case of hesperidin FH1 is having the minimum drug entrapment efficiency of approximately $69.46 \%$ whereas the entrapment efficiency of FH5 shows an approximate entrapment of $74.28 \%$. Drug: sodium alginate ratio of $1: 2$ shows an approximate entrapment of $74.28 \%$. In case of Rutin FR1 is having the minimum drug entrapment efficiency of approximately $71.00 \%$ whereas the entrapment efficiency of FR2 shows an approximate entrapment of $75.7 \%$. This could be revealed as the increase in polymer concentration has significant effect on the drug entrapment efficiency. Drug: sodium slginate ratio of 1:1.2 shows an approximate entrapment of $75.7 \%$.

In vitro release study: In vitro release studies of the prepared micro particles containing the drugs were carried out using USP Type-II dissolution apparatus of rotating paddle type. The dissolution medium used for this purpose was phosphate buffer pH 6.8. Samples were collected at specific time period and the concentration of drug present in it was measured spectrophotometrically. Alternatively fresh fluid was added

\begin{tabular}{cccc}
\multicolumn{4}{c}{ TABLE-2 } \\
& $\begin{array}{c}\text { DRUG ENTRAPPMENT EFFICIENCY } \\
\text { (RUTIN AND HESPIRIDIN) }\end{array}$ \\
\hline $\begin{array}{c}\text { Formulation } \\
\text { code (rutin) }\end{array}$ & $\begin{array}{c}\text { Drug } \\
\text { content (\%) }\end{array}$ & $\begin{array}{c}\text { Formulation code } \\
\text { (hesperidin) }\end{array}$ & $\begin{array}{c}\text { Drug } \\
\text { content (\%) }\end{array}$ \\
\hline FH 1 & 69.46 & FR1 & 71.0 \\
FH 2 & 73.60 & FR2 & 75.7 \\
FH 3 & 71.50 & FR3 & 74.5 \\
FH 4 & 70.36 & FR4 & 74.9 \\
FH 5 & 74.28 & FR5 & 74.0 \\
\hline
\end{tabular}

to maintain the sink condition. Results for release study of drugs from the formulations are shown in Table- 3 . The results of the dissolution study indicated that the amount of drug release appreciably decreased and became steady with an increase in the concentration of sodium alginate in the matrix. It can be ascribed to increase in the densities of the polymer matrix ensuing in larger microspheres and this in turn increases the diffusional path length, which the drug molecules have to traverse as supported by the preceding work of Sultana et al. [16] in preparation of diltiazem microspheres.

Drug release kinetic studies: The drug release kinetic studies was studied by using different models like zero order, first order, Higuchi plot and Krosmayer Peepas models. The regression coefficient was found out in each model. The release kinetics of the prepared batches of formulation found that the prepared batches followed Higuchi and Krosmayer Peepas release mechanism.

Scanning electron microscopy: The SEM photo-micrograph shown in Figs. 2 and 3 was the images of drug loaded microspheres of formulations in 50X and 100X, respec-tively. The microspheres were almost spherical in shape with rough and non-porous surface.

\section{Conclusion}

Rutin and hesperidin appears to have limited therapeutic window through oral route, which is demanding to produce an ideal delivery technique to increase the therapeutic concentration in vivo. The present study has shown that sodium alginate coated microspheres could be a challenging controlled drug delivery system for rutin and hesperidin with more therapeutic bioavailability. Hence, it can be concluded that using these cost effective polymers can develop a novel drug delivery

TABLE-3

ESTIMATION OF \% CUMULATIVE DRUG RELASE FOR in vitro DRUG RELEASE STUDY OF THE FORMULATIONS

\begin{tabular}{|c|c|c|c|c|c|c|c|c|c|c|}
\hline \multicolumn{5}{|c|}{ Cumulative drug release $(\%)$} & \multirow{4}{*}{$\begin{array}{l}\text { Time } \\
\text { (h) }\end{array}$} & \multicolumn{5}{|c|}{ Cumulative drug release $(\%)$} \\
\hline \multicolumn{5}{|c|}{ Hesperidin } & & \multicolumn{5}{|c|}{ Rutin } \\
\hline FH1 & $\mathrm{FH} 2$ & FH3 & FH4 & FH5 & & FR1 & FR2 & FR3 & FR4 & FR5 \\
\hline $1: 1$ & 1.1 .12 & $1: 1.25$ & $1: 1.5$ & 1: 2 & & $1: 1$ & 1.1 .12 & $1: 1.25$ & $1: 1.5$ & 1: 2 \\
\hline 47.52 & 42.44 & 37.00 & 34.90 & 28.20 & 1 & 44.61 & 41.99 & 39.66 & 36.20 & 32.14 \\
\hline 56.87 & 47.26 & 44.10 & 39.29 & 36.26 & 2 & 54.46 & 49.20 & 45.21 & 41.15 & 40.67 \\
\hline 69.12 & 63.11 & 58.20 & 53.46 & 39.43 & 3 & 62.56 & 57.43 & 52.20 & 47.50 & 43.89 \\
\hline 72.45 & 69.45 & 62.10 & 60.59 & 46.20 & 4 & 79.40 & 65.56 & 59.56 & 54.66 & 47.92 \\
\hline 86.32 & 73.61 & 69.20 & 66.23 & 52.32 & 5 & 83.46 & 77.68 & 64.66 & 68.49 & 51.02 \\
\hline 91.29 & 88.89 & 73.01 & 51.66 & 48.60 & 6 & 99.69 & 80.66 & 78.18 & 74.50 & 64.11 \\
\hline 106.09 & 94.29 & 81.33 & 79.66 & 65.65 & 7 & 108.20 & 95.45 & 82.20 & 79.33 & 79.65 \\
\hline- & 99.66 & 96.66 & 82.20 & 79.32 & 8 & - & 109.56 & 87.22 & 85.32 & 83.50 \\
\hline- & - & 99.69 & 96.20 & 83.23 & 9 & - & - & 92.23 & 89.69 & 85.20 \\
\hline- & - & - & 100.20 & 99.30 & 10 & - & - & 99.60 & 94.70 & 90.23 \\
\hline- & - & - & - & 102.30 & 12 & - & - & - & 100.20 & 107.67 \\
\hline
\end{tabular}




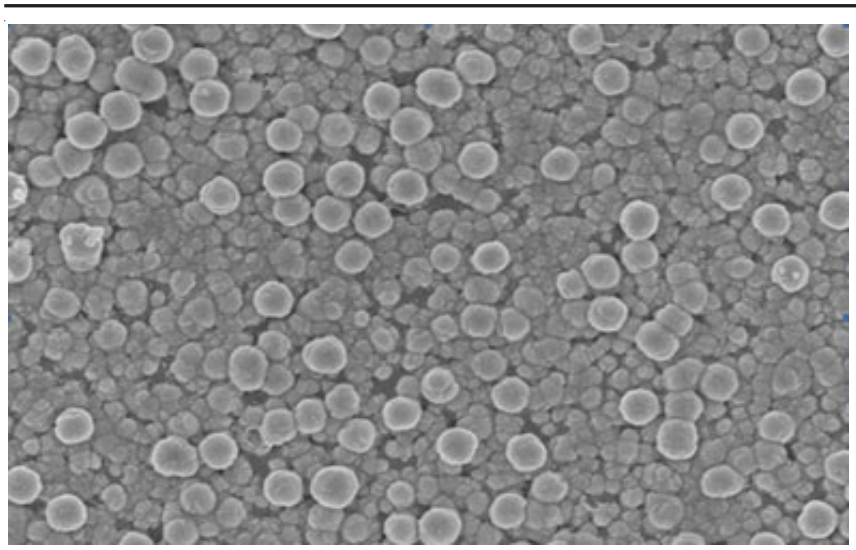

Fig. 2. SEM image at 50X magnification

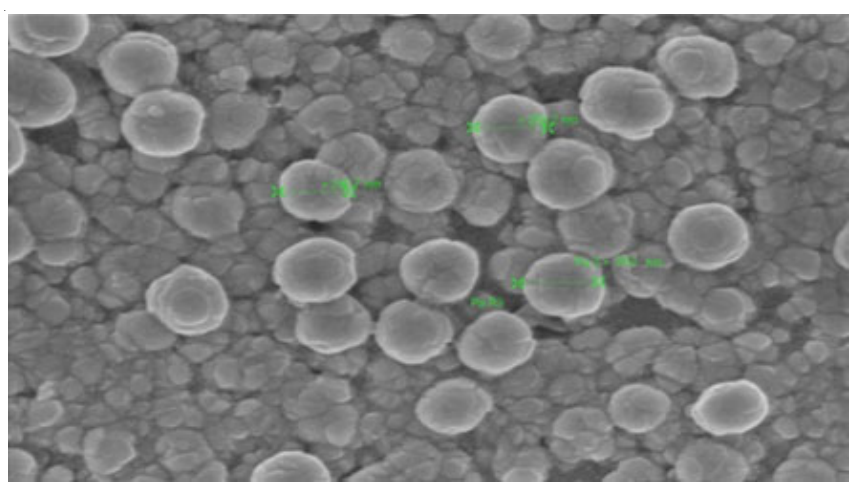

Fig. 3. SEM image at $100 \mathrm{X}$ magnification

system of herbal drug rutin and hesperidin which can increase the bioavailability of the compound for several degenerative diseases.

\section{REFERENCES}

1. P. Ganesan, A.J.D. Johnson, L. Sabapathy and A. Duraikannu, Am. J. Drug Discov. Develop., 4, 153 (2014); https://doi.org/10.3923/ajdd.2014.153.179.

2. A. Kundu and S. Datta, Int. J. Adv. Pharm. Biol. Chem., 1, 266 (2012).

3. P.G. Pietta, J. Nat. Prod., 63, 1035 (2000); https://doi.org/10.1021/np9904509.

4. S. Burda and W. Oleszek, J. Agric. Food Chem., 49, 2774 (2001); https://doi.org/10.1021/jf001413m.

5. H. Javed, M.M. Khan, A. Ahmad, K. Vaibhav, M.E. Ahmad, A. Khan, M. Ashafaq, F. Islam, M.S. Siddiqui, M.M. Safhi and F. Islam, Neuroscience, 210, 340 (2012); https://doi.org/10.1016/j.neuroscience.2012.02.046.

6. M. Nassiri-Asl, S.R. Mortazavi, F. Samiee-Rad, A.A. Zangivand, F. Safdari, S. Saroukhani and E. Abbasi, Epilepsy Behav., 18, 50 (2010); https://doi.org/10.1016/j.yebeh.2010.03.005.

7. G.B. Reddy, P. Muthenna, C. Akileshwari, M. Saraswat and J.M. Petrash, Curr. Sci., 101, 1191 (2011)

8. O. Sipahigil and B. Dortunc, Int. J. Pharm., 1, 86 (2004).

9. A. Lohumi, S. Rawat, S. Sarkar, A.B. Sipai and V.N. Yadav, J. Drug Deliv. Therap., 2, 129 (2012).

10. S. De, R. Kundu and A. Biswas, J. Colloid Interface Sci., 386, 9 (2012); https://doi.org/10.1016/j.jcis.2012.06.073.

11. L. Tavano, P. Alfano, R. Muzzalupo and B. de Cindio, Colloids Surf. B, 87, 333 (2011); https://doi.org/10.1016/j.colsurfb.2011.05.041.

12. S. Badhana, A. Garud and A. Garud, Int. Curr. Pharm. J., 2, 42 (2013); https://doi.org/10.3329/icpj.v2i3.13577.

13. A.R. Tapas, D.M. Sakarkar and R.B. Kakde, Trop. J. Pharm. Res., 7, 1089 (2008); https://doi.org/10.4314/tjpr.v7i3.14693.

14. N.D. Paladugu and B. Satyanarayana, Int. J. Pharm. Chem. Biol. Sci., 3, 586 (2013)

15. J. Zhang, S. Xu, S. Zhang and Z. Du, Iran. Polym. J., 17, 899 (2008).

16. Y. Sultana, S. Mall, D.P. Maurya, D. Kumar and M. Das, Pharm. Dev. Technol., 14, 321 (2009); https://doi.org/10.1080/10837450802626304. 